\title{
Classical pseudotenors and positivity in small regions
}

\author{
${ }^{1}$ Lau Loi So, ${ }^{2}$ James M. Nester and ${ }^{3}$ Hsin Chen \\ ${ }^{1,3}$ Department of Physics, National Central University, Chung-Li 320, Taiwan. \\ ${ }^{2}$ Dept. of Physics and Institute of Astronomy, National Central University, \\ Chung-Li 320, Taiwan.
}

\begin{abstract}
We have studied the famous classical pseudotensors in the small region limit, both inside matter and in vacuum. A recent work [Deser et al. 1999 CQG 16, 2815] had found one combination of the Einstein and Landau-Lifshitz expressions which yields the Bel-Robinson tensor in vacuum. Using similar methods we found another independent combination of the Bergmann-Thomson, Papapetrou and Weinberg pseudotensors with the same desired property. Moreover we have constructed an infinite number of additional new holonomic pseudotensors satisfying this important positive energy requirement, all seem quite artificial. On the other hand we found that Møller's 1961 tetrad-teleparallel energy-momentum expression naturally has this Bel-Robinson property.
\end{abstract}

\section{Introduction: quasilocal quantities for small re- gions}

The localization of gravitational energy-momentum remains an important problem in GR. Using standard methods many famous researchers each found their own expression. None of these expressions is covariant, they are all reference frame dependent (referred to as "pseudotensors"). This feature can be understood in terms of the equivalence principle: gravity cannot be detected at a point, so it cannot have a point-wise defined energy-momentum density. Now there is another way to address the difficulty.

The new idea is quasilocal: energy-momentum is associated with a closed 2 surface surrounding a region [1. A good quasilocal approach is in terms of the Hamiltonian 2]. Then the Hamiltonian boundary term determines the quasilocal quantities. In fact this approach includes all the traditional pseudotensors [3, 4]. They are each generated by a superpotential which can serve as special type of Hamiltonian boundary term.

A good energy-momentum expression for gravitating systems should satisfy a variety of requirements, including giving the standard values for the total quantities for 
asymptotically flat space and reducing to the material energy-momentum in the appropriate limit. No entirely satisfactory expression has yet been identified. One of the most restrictive requirements is positivity. A general positivity proof is very difficult. One limit that is not so difficult is the small region limit. This has not previously been systematically investigated for all the classical pseudotensors expressions. In matter the expression should be dominated by the material energy-momentum tensor. In vacuum we require that its Taylor series expansion in Riemann normal coordinates have at the second order a positive multiple of the Bel-Robinson tensor. This will guarantee positive energy in small vacuum regions.

\section{The classical pseudotensors}

Recall the Einstein field equation $G_{\mu \nu}=R_{\mu \nu}-\frac{1}{2} g_{\mu \nu} R=\kappa T_{\mu \nu}$. Matter energymomentum has a vanishing covariant divergence $\nabla_{\mu} T^{\mu}{ }_{\nu}=0$, but in curved spacetime this is not in the form of a conserved energy-momentum relation. But one can rewrite it in the form of a divergence

$$
\partial_{\mu} \sqrt{-g}\left(T_{\nu}^{\mu}+t^{\mu}{ }_{\nu}\right)=0
$$

here $t^{\mu}{ }_{\nu}$ is the gravitational energy-momentum pseudotensor. The energy-momentum complex $\mathcal{T}^{\mu}{ }_{\nu}$ is then given as

$$
\kappa \mathcal{T}^{\mu}{ }_{\nu}=\kappa \sqrt{-g}\left(T^{\mu}{ }_{\nu}+t^{\mu}{ }_{\nu}\right) \equiv \partial_{\lambda} U_{\nu}{ }^{[\mu \lambda]},
$$

where $U_{\nu}^{[\mu \lambda]}$ is called the superpotential.

\section{Riemann normal coordinates and the adapted tetrads}

For the total quantities of an isolated gravitating system the various expressions give the expected weak field asymptotic values. However they are quite different in the strong field region. To study the quasilocal quantities one can Taylor expand the Hamiltonian, including the divergence of its boundary term, in a small spatial region surrounding a point. The reference is the flat space geometry at this origin. Riemann normal coordinates (RNC) satisfy

$$
g_{\mu \nu}(0)=\eta_{\mu \nu}, \quad \partial_{\lambda} g_{\mu \nu}(0)=0, \quad \partial^{2}{ }_{\mu \nu} g_{\alpha \beta}(0)=-\frac{1}{3}\left(R_{\alpha \mu \beta \nu}+R_{\alpha \nu \beta \mu}\right)(0) .
$$


Later we will also need the associated adapted orthonormal frame (aka tetrad, vierbein) which satisfies $g_{\mu \nu}=\eta_{a b} e^{a}{ }_{\mu} e^{b}{ }_{\nu}$ and

$$
e_{\mu}^{a}(0)=\delta_{\mu}^{a}, \quad \partial_{\nu} e_{\nu}^{a}(0)=0, \quad \Gamma_{b \mu}^{a}(0)=0, \quad \partial_{\mu} \Gamma_{b \nu}^{a}(0)=\frac{1}{2} R_{b \mu \nu}^{a}(0) .
$$

For the energy-momentum density we want at non-vacuum points the results to be dominated by the material energy-momentum. At vacuum points one wants a result proportional to the Bel-Robinson tensor. That will guarantee the proper positive energy property; i.e. the associated energy-momentum vector will then be future pointing and non-space like.

\section{Quadratic curvature combinations}

The Bel-Robinson tensor $B_{\alpha \beta \mu \nu}$ and the tensors $S_{\alpha \beta \mu \nu}, K_{\alpha \beta \mu \nu}$ and $T_{\alpha \beta \mu \nu}$ are defined as follows

$$
\begin{aligned}
B_{\alpha \beta \mu \nu} & :=R_{\alpha \lambda \mu \sigma} R_{\beta}{ }^{\lambda}{ }_{\nu}{ }^{\lambda}+R_{\alpha \lambda \nu \sigma} R_{\beta}{ }^{\lambda}{ }_{\mu}{ }^{\prime}+3 T_{\alpha \beta \mu \nu}, \\
S_{\alpha \beta \mu \nu} & :=R_{\alpha \mu \lambda \sigma} R_{\beta \nu}{ }^{\lambda \sigma}+R_{\alpha \nu \lambda \sigma} R_{\beta \mu}{ }^{\lambda \sigma}-6 T_{\alpha \beta \mu \nu}, \\
K_{\alpha \beta \mu \nu} & :=R_{\alpha \lambda \mu \sigma} R_{\beta}{ }^{\lambda}{ }_{\nu}{ }^{\sigma}+R_{\alpha \lambda \nu \sigma} R_{\beta}{ }^{\lambda}{ }_{\mu}{ }+9 T_{\alpha \beta \mu \nu}, \\
T_{\alpha \beta \mu \nu} & :=-\frac{1}{24} g_{\alpha \beta} g_{\mu \nu} R_{\lambda \sigma \xi \kappa} R^{\lambda \sigma \xi \kappa} .
\end{aligned}
$$

A recent work [5] had found exactly one pseudotensor expression, a certain combination of the Einstein and Landau-Lifshitz expressions, which yields the Bel-Robinson tensor in vacuum. They argued that this combination is unique under their assumptions.

\section{Classical holonomic pseudotensors}

The well known classical superpotentials associated with the Einstein, Landau-Lifshitz, Bergmann-Thomson, Papapetrou, Weinberg and Møller(1958) energy-momentum complexes are

$$
\begin{aligned}
{ }_{E} U_{\alpha}^{[\mu \nu]} & =\sqrt{-g} g^{\beta \sigma} \Gamma^{\tau}{ }_{\lambda \beta} \delta_{\tau \sigma \alpha}^{\lambda \nu \mu}, \\
{ }_{B} U^{\alpha[\mu \nu]} & =\sqrt{-g} g^{\alpha \beta} g^{\pi \sigma} \Gamma_{\lambda \pi}^{\tau} \delta_{\tau \sigma \beta}^{\lambda \nu \mu}=\sqrt{-g} U_{L}^{\alpha[\mu \nu]}, \\
{ }_{P} H^{[\mu \nu][\alpha \beta]} & =\sqrt{-g} g^{m a} g^{n d} \delta_{a b}^{\mu \nu} \delta_{m n}^{\alpha \beta},
\end{aligned}
$$




$$
\begin{aligned}
{ }_{W} H^{[\mu \alpha][\nu \beta]} & =\sqrt{-\eta}\left(\eta^{m c} \eta^{n d}-\frac{1}{2} \eta^{m n} \eta^{c d}\right) g_{c d} \eta^{a b} \delta_{m a}^{\alpha \mu} \delta_{n b}^{\nu \beta}, \\
{ }_{58} U_{\alpha}{ }^{[\mu \nu]} & =\sqrt{-g}\left(\Gamma^{\nu \mu}{ }_{\alpha}-\Gamma^{\mu \nu}{ }_{\alpha}\right) .
\end{aligned}
$$

The pseudotensors are obtained according to one of the prescriptions:

$$
\mathcal{T}_{\alpha}{ }^{\mu}=\partial_{\nu} U_{\alpha}{ }^{[\mu \nu]}, \quad \mathcal{T}^{\alpha \mu}=\partial_{\nu} U^{\alpha[\mu \nu]}, \quad \mathcal{T}^{\mu \nu}=\partial^{2}{ }_{\alpha \beta} H^{[\mu \alpha][\nu \beta]} .
$$

Inside matter at the origin, the RNC expansion results are

$$
E_{\alpha}^{\beta}(0)=B_{\alpha}^{\beta}(0)=P_{\alpha}^{\beta}(0)=W_{\alpha}^{\beta}(0)=2 G_{\alpha}^{\beta}(0)=2 \kappa T_{\alpha}^{\beta}(0) .
$$

This is as expected from the equivalence principle. However the Møller(1958) expression gives

$$
M_{\alpha}^{\beta}(0)=R_{\alpha}^{\beta}(0)=\kappa\left(T_{\alpha}^{\beta}-\frac{1}{2} \eta_{\alpha}{ }^{\beta} T\right)(0) \neq 2 \kappa T_{\alpha}^{\beta}(0) .
$$

This result is not acceptable. From [5], the small vacuum $\left(G_{\mu \nu}=0\right)$ region nonvanishing terms are

$$
\begin{aligned}
E_{\alpha}{ }^{\beta} & =-2 \Gamma_{\lambda \sigma \alpha} \Gamma^{\beta \lambda \sigma}+\delta_{\alpha}^{\beta} \Gamma_{\lambda \sigma \tau} \Gamma^{\tau \lambda \sigma} \\
L^{\alpha \beta} & =\Gamma_{\lambda \sigma}^{\alpha}\left(\Gamma^{\beta \lambda \sigma}-\Gamma^{\lambda \sigma \beta}\right)-\Gamma_{\lambda \sigma}^{\alpha}\left(\Gamma^{\beta \lambda \sigma}+\Gamma^{\sigma \lambda \beta}\right)+g^{\alpha \beta} \Gamma_{\lambda \sigma \tau} \Gamma^{\sigma \lambda \tau}
\end{aligned}
$$

Using a similar technique we found, for the other pseudotensors (note Papapetrou and Weinberg depend explicitly on the Minkowski metric $\left.\eta_{\alpha \beta}\right)$,

$$
\begin{aligned}
P^{\alpha \beta} & =L^{\alpha \beta}+h^{\lambda \sigma}\left(\Gamma_{\lambda, \sigma}^{\alpha \beta}+\Gamma_{\lambda, \sigma}^{\beta \alpha}{ }_{\lambda{ }_{, \sigma}}\left(\Gamma_{\lambda}^{\alpha \sigma}+h_{\lambda}^{\lambda \beta} \Gamma_{\lambda}^{\sigma \alpha}\right),\right. \\
W^{\alpha \beta} & =-2 \Gamma_{\lambda \sigma}{ }^{\alpha} \Gamma^{\lambda \sigma \beta}+g^{\alpha \beta} \Gamma_{\lambda \sigma \tau} \Gamma^{\lambda \sigma \tau}-g^{\alpha \lambda} g^{\beta \pi} h^{\sigma \rho} \delta_{\lambda \sigma}^{c k} \delta_{\pi \rho}^{\xi d}\left(\Gamma_{d c \kappa, \xi}+\Gamma_{c d \kappa, \xi}\right), \\
M_{\alpha}{ }^{\beta} & =\Gamma_{\lambda \sigma \alpha} \Gamma^{\lambda \sigma \beta}-\Gamma_{\lambda \sigma \alpha} \Gamma^{\sigma \lambda \beta}+\left(g^{\beta \sigma} g^{\psi \lambda}-g^{\beta \psi} g^{\lambda \sigma}\right) g_{\alpha \psi, \lambda \sigma} .
\end{aligned}
$$

Here $h_{\alpha \beta}:=g_{\alpha \beta}-\eta_{\alpha \beta}$ and $h_{\alpha \beta}=-\frac{1}{3} R_{\alpha \lambda \beta \sigma} x^{\lambda} x^{\sigma}+O\left(x^{3}\right)$ in RNC. From [5] in vacuum we have

$$
\partial_{\mu \nu}^{2} E_{\alpha \beta}(0)=\frac{1}{9}\left(4 B_{\alpha \beta \mu \nu}-S_{\alpha \beta \mu \nu}\right), \quad \partial_{\mu \nu}^{2} L_{\alpha \beta}(0)=\frac{1}{9}\left(7 B_{\alpha \beta \mu \nu}+\frac{1}{2} S_{\alpha \beta \mu \nu}\right) .
$$

In order to obtain the Bel-Robinson tensor, Deser et al. used a "by hand" combination

$$
\partial_{\mu \nu}^{2}\left(\frac{1}{2} E_{\alpha \beta}+L_{\alpha \beta}\right)=B_{\alpha \beta \mu \nu},
$$


Using similar methods we obtained for the other pseudotensors in vacuum at the origin

$$
\begin{aligned}
\partial_{\mu \nu}^{2} P_{\alpha \beta}(0) & =\frac{2}{9}\left(4 B_{\alpha \beta \mu \nu}-S_{\alpha \beta \mu \nu}-K_{\alpha \beta \mu \nu}\right) \\
\partial_{\mu \nu}^{2} W_{\alpha \beta}(0) & =-\frac{2}{9}\left(B_{\alpha \beta \mu \nu}+2 S_{\alpha \beta \mu \nu}+3 K_{\alpha \beta \mu \nu}\right) \\
\partial_{\mu \nu}^{2} M_{\alpha \beta}(0) & =\frac{1}{9}\left(2 B_{\alpha \beta \mu \nu}-\frac{1}{2} S_{\alpha \beta \mu \nu}-K_{\alpha \beta \mu \nu}\right) .
\end{aligned}
$$

(Here we have included for completeness the result of the Møller(1958) expression, even though it does not have a good matter interior limit.) From this we find one more independent combination of the Landau-Lifshitz, Papapetrou and Weinberg pseudotensors with the same desired Bel-Robinson property. (Note: the earlier work cited above did not get this result, as they had excluded the explicit use of the Minkowski metric in the superpotentials they considered.) Inside matter and in vacuum at the origin, respectively, we find

$$
\frac{1}{3}\left[2 L_{\alpha \beta}+\frac{1}{2}\left(3 P_{\alpha \beta}-W_{\alpha \beta}\right)\right](0)=2 G_{\alpha \beta}(0), \quad \frac{1}{3} \partial^{2}{ }_{\mu \nu}\left[2 L_{\alpha \beta}+\frac{1}{2}\left(3 P_{\alpha \beta}-W_{\alpha \beta}\right)\right](0)=B_{\alpha \beta \mu \nu .} .
$$

\section{A large class of new pseudotensors}

Moreover we have constructed an infinite number (a 3 parameter set) of new holonomic pseudotensors all satisfying this important Bel-Robinson/positivity property. The new general superpotential is

$$
\mathcal{U}_{\alpha}{ }^{[\mu \nu]}={ }_{E} U_{\alpha}{ }^{[\mu \nu]}+\left\{\begin{array}{c}
c_{1} h^{\mu \pi} \Gamma_{\alpha}{ }^{\nu}{ }_{\pi}+c_{2} h^{\mu \pi} \Gamma^{\nu}{ }_{\alpha \pi}+c_{3} h^{\mu \pi} \Gamma_{\pi}{ }^{\nu}{ }_{\alpha} \\
+c_{4}^{\prime} \delta_{\alpha}^{\mu} h^{\pi \rho} \Gamma^{\nu}{ }_{\pi \rho}+c_{4}^{\prime \prime} \delta_{\alpha}^{\mu} h^{\pi \rho} \Gamma_{\pi}{ }^{\nu}{ }_{\rho}+c_{5} h_{\alpha \pi} \Gamma^{\mu \nu \pi}-(\nu \leftrightarrow \mu)
\end{array}\right\},
$$

where $c_{1}$ to $c_{5}$ are constants and $h_{\alpha \beta}:=g_{\alpha \beta}-\eta_{\alpha \beta}$. In Riemann normal coordinates $h^{\alpha \beta}=\frac{1}{3} R^{\alpha}{ }_{\xi}{ }_{\kappa} x^{\xi} x^{\kappa}+O\left(x^{3}\right)$. Actually, the leading superpotential ${ }_{E} U_{\alpha}{ }^{[\mu \nu]}$ in (28) is not necessary Freud's, it can be replaced by any other which offers a good spatially asymptotic and small region material limit. The resultant energy density inside matter at the origin is, as expected,

$$
2 \kappa \mathcal{E}_{\alpha}{ }^{\beta}(0)=2 G_{\alpha}{ }^{\beta}(0)=2 \kappa T_{\alpha}^{\beta}(0) .
$$


The RNC second derivatives in vacuum at the origin are

$$
\partial_{\mu \nu}^{2} \mathcal{E}_{\alpha \beta}(0)=\frac{1}{9}\left\{\begin{array}{c}
\left(4-2 c_{1}+c_{2}+c_{3}-4 c_{4}+3 c_{5}\right) B_{\alpha \beta \mu \nu} \\
-\frac{1}{2}\left(2-c_{1}-4 c_{2}+5 c_{3}-2 c_{4}-3 c_{5}\right) S_{\alpha \beta \mu \nu} \\
+\left(c_{1}+c_{2}-2 c_{3}-2 c_{4}\right) K_{\alpha \beta \mu \nu}
\end{array}\right\} .
$$

where $c_{4}=c_{4}^{\prime}-\frac{1}{2} c_{4}^{\prime \prime}$. Note that we can choose

$$
\begin{array}{r}
4-2 c_{1}+c_{2}+c_{3}-4 c_{4}+3 c_{5}>0, \\
2-c_{1}-4 c_{2}+5 c_{3}-2 c_{4}-3 c_{5}=0, \\
c_{1}+c_{2}-2 c_{3}-2 c_{4}=0 .
\end{array}
$$

The solutions can be parameterized as follows,

$c_{1}+c_{2}-2 c_{3}<1, \quad\left(c_{1}, c_{2}, c_{3}, c_{4}, c_{5}\right)=\left(c_{1}, c_{2}, c_{3}, \frac{1}{2}\left(c_{1}+c_{2}+2 c_{3}\right), \frac{1}{3}\left(2-2 c_{1}-5 c_{2}+7 c_{3}\right)\right)$.

Then the second derivatives of the new pseudotensors in vacuum are

$$
\partial_{\mu \nu}^{2} \mathcal{E}_{\alpha \beta}(0)=\frac{2}{3}\left(1-c_{1}-c_{2}+2 c_{3}\right) B_{\alpha \beta \mu \nu} .
$$

As there are three arbitrary constants that one can tune, obviously there exists an infinite number of solutions with any positive magnitude of $B_{\alpha \beta \mu \nu}$. They all appear to be highly artificial. It seems that there is no obstruction in going to higher order. From our analysis we infer that there are an infinite number of holonomic gravitational energy-momentum pseudotensor expressions which satisfy the highly desired small region Bel-Robinson/positive energy property.

\section{Møller's 1961 tetrad-teleparallel energy-momentum tensor}

On the other hand, unlike the aforementioned mathematically and physically contrived expressions, we found that Møller's 1961 teleparallel-tetrad energy-momentum expression naturally has the desired Bel-Robinson property. The superpotential has the same form as Freud's but the indices now refer to a tetrad:

$$
{ }_{61} U_{a}^{[b c]}=\sqrt{-g} g^{d f} \Gamma_{f e}^{i} e_{i d a}^{b c e} .
$$


Expressed in terms of differential forms we have

$$
m_{a}{ }^{b} \eta_{b}=d\left(\Gamma^{b}{ }_{c} \wedge \eta_{b}^{c}{ }_{a}^{c}\right) \text {. }
$$

The RNC and adapted frame expansion results inside matter, and the vacuum second derivatives at the origin, respectively, are

$$
m_{a}{ }^{b}(0)=2 G_{a}{ }^{b}(0)=2 \kappa T_{a}{ }^{b}(0), \quad \partial_{\mu \nu}^{2} m_{a b}(0)=\frac{1}{2} B_{a b \mu \nu} .
$$

Thus the desired Bel-Robinson property is naturally satisfied. An important consequence is that the gravitational energy according to this measure is positive, at least to this order. (We expected this positivity result since in fact Møller's 1961 expression has an associated positive energy proof [6].) Once again Møller's 1961 tensor stands out as one of the best descriptions for gravitational energy-momentum.

\section{References}

[1] L. $\quad$ B. Szabados, Living Rev. Relativity, $7 \quad$ (2004) 4; http://www.livingreviews.org/lrr-2004-4

[2] C. M. Chen and J.M. Nester, Class. Quantum Grav. 16 (1999) 1279 arXiv:gr-qc/9809020.

[3] C. C. Chang, J. M. Nester and C. M. Chen, Phys. Rev. Lett. 83 (1999) 1897.

[4] C. M. Chen and J. M. Nester, Grav. \& Cosmol. 6 (2000) 257 arXiv:gr-qc/0001088.

[5] S. Deser, J. S. Franklin and D. Seminara, Class. Quantum Grav. 16 (1999) 2815.

[6] J. M. Nester, Int. J. Mod. Phys. A. 4 (1989) 1755. 